\title{
Coherence and noise structure of the octave-spanning IR supercontinua
}

\author{
Vladimir L. Kalashnikov, Evgeni Sorokin, Irina T. Sorokina \\ Institut fuer Photonik, TU Wien, Gusshausstr. 27/387, A-1040 Vienna, Austria \\ e-mail:kalashnikov@tuwien.ac.at
}

\begin{abstract}
Noise structure and coherence of mid-IR supercontinua in fibers with strong Raman nonlinearity were investigated. It was established that the octave-spanning spectra retain very high degree of coherence in the presence of the enhanced nonlinearity.

(C)2006 Optical Society of America

OCIS codes: (190.4370) Nonlinear optics, fibers; (320.7140) Ultrafast processes in fibers
\end{abstract}

Photonic crystal fibers (PCFs) provide extremely broad supercontinuum (SC) generation at sub-nJ input pulse energies in the near-infrared wavelength range. This was possible due to the mechanism of the so-called high-order soliton fission in the vicinity of zero-dispersion wavelength [1]. However, this mechanism loses its efficiency in IR wavelength range. This results from the decrease of the effective electronic nonlinearity, which scales approximately as $\propto 1 / \lambda^{3}$ [2]. Therefore, in order to provide more than octave-spanning SC, it is necessary to use the highnonlinear fibers like SF6 [3,4] pumped by the femtosecond pulses from the fiber or Cr:YAG laser [2].

Our Raman spectrum measurements have shown that such fibers (SF6 and SF57) possess higher Raman gain and twice higher Raman frequency shift in comparison with that in fused silica [5]. This can provide an addition spectral extra-broadening in mid-IR, but can also affect the coherence and the noise structure of SC.

The aim of this paper was to investigate the coherence of octave-spanning IR SC from the highly-nonlinear PCFs and to demonstrate that the enhanced nonlinearity (in particular, stimulated Raman scattering (SRS)) does not reduce the SC coherence. Our treatment was based on the Wigner phase-space representation of the quantized field evolution in fiber [6]:

$$
\frac{\partial}{\partial z} \psi(t, z)=i \sum_{n=2}^{N} \frac{i^{n} \beta_{n}}{n !} \frac{\partial^{n}}{\partial t^{n}} \psi(t, z)+i\left\{\gamma \psi \psi^{+}+\int_{-\infty}^{t} h(t-\tau) \psi \psi^{+} d \tau\right\} \psi(t, z)+i \Gamma(t)+\text { h.c. },
$$

where we omitted the phase-term and h.c. means the hermite-conjugated equation for $\psi^{+} . \psi(t, z)$ is proportional to slowly varying time- $(t)$ and propagation distance- $(z)$ dependent field envelope; $\beta_{n}$ is the $n$-order group-delay dispersion of glass; $\gamma$ is the Kerr nonlinear coefficient ( $\psi \psi^{+}$is proportional to the field intensity); $h$ is the Raman response function. $\Gamma$ is the noise term including the contribution of i) input quantum noise, fluctuating ii) pump pulse power, iii) duration, iv) carrier frequency, v) timing, and vi) Raman noise.

The dependences of the averaged spectral powers on the pump pulse intensity for two types of the highlynonlinear fibers are shown in Fig.1. SRS causes the threshold-like extra-broadening at 7.5 and $5 \mathrm{GW} / \mathrm{cm}^{2}$ pump intensities for SF6 and SF57 fibers, respectively. Both fibers operate in the vicinity of zero-dispersion wavelength. Large Raman shift $\left(\approx 1000 \mathrm{~cm}^{-1}\right)$ allows the octave-spanning spectra for $<200 \mathrm{pJ}$ pump energies. However, according to previous investigations such a shift may reduce the spectral coherence [7]. In order to study the spectrum coherence, we simulated the field propagation on the basis of the above described stochastic equation for 128 noised pump pulses. The resulted spectral coherence is shown in Fig. 2. It was found that the enhanced SRS does not reduce the coherence. We suppose that this results from an essentially reduced threshold of the spectral extrabroadening. Simulations demonstrated that the noise level essentially reduces inside the spectrum and contributes only within the spectral "gaps". Hence, these fibers have been highly attractive for the noise-less octave-spanning IR $\mathrm{SC}$ generation with considerably reduced thresholds $(<200 \mathrm{pJ})$.

The research was supported by the Austrian FWF Funds (project P17973). 


\section{JWB29.pdf}
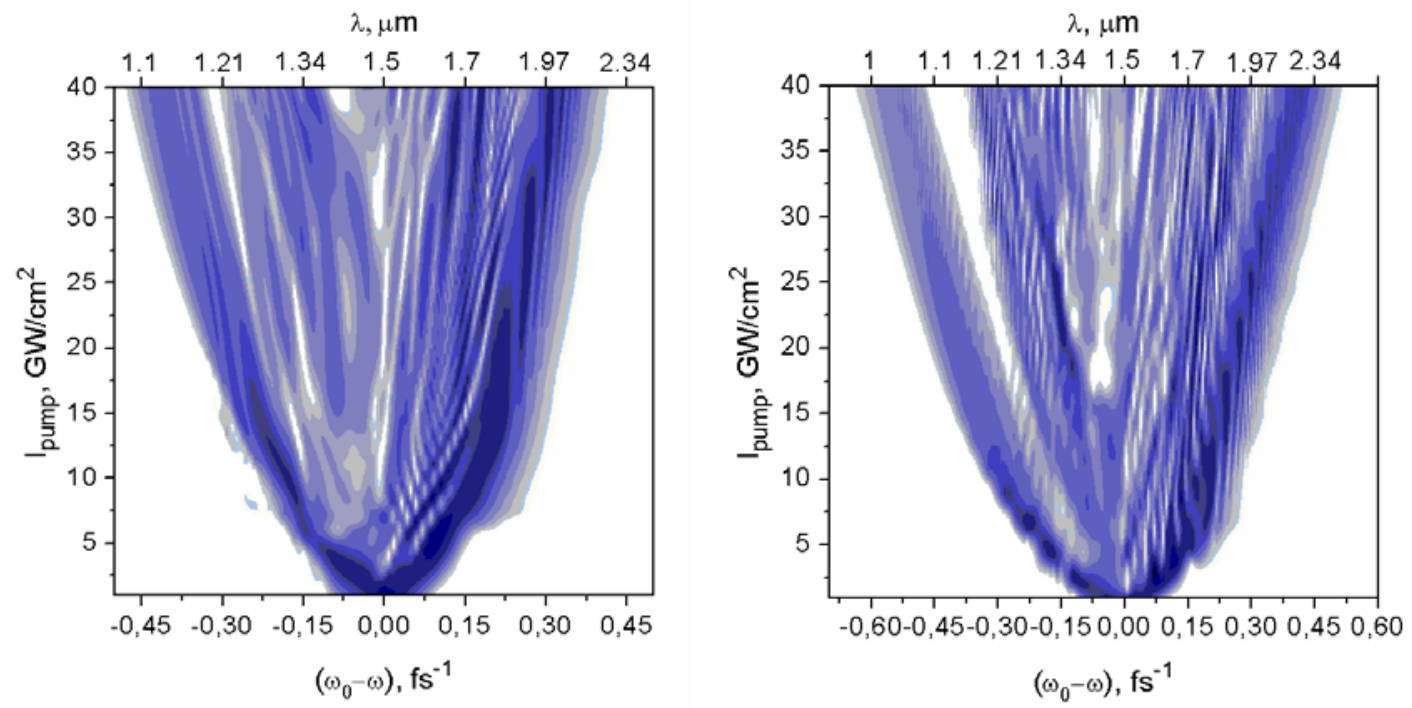

Fig. 1. Logarithm of spectral power (from -30dB level) as a function of the input intensity of the 60 fs pump pulse from Cr:YAG laser operating at $1.5 \mu \mathrm{m}$ wavelength. Left graph: SF6 fiber with $2.6 \mu \mathrm{m}$ core radius. Right graph: SF57 fiber with $2.25 \mu \mathrm{m}$ core radius. Propagation length is $20 \mathrm{~cm}$.

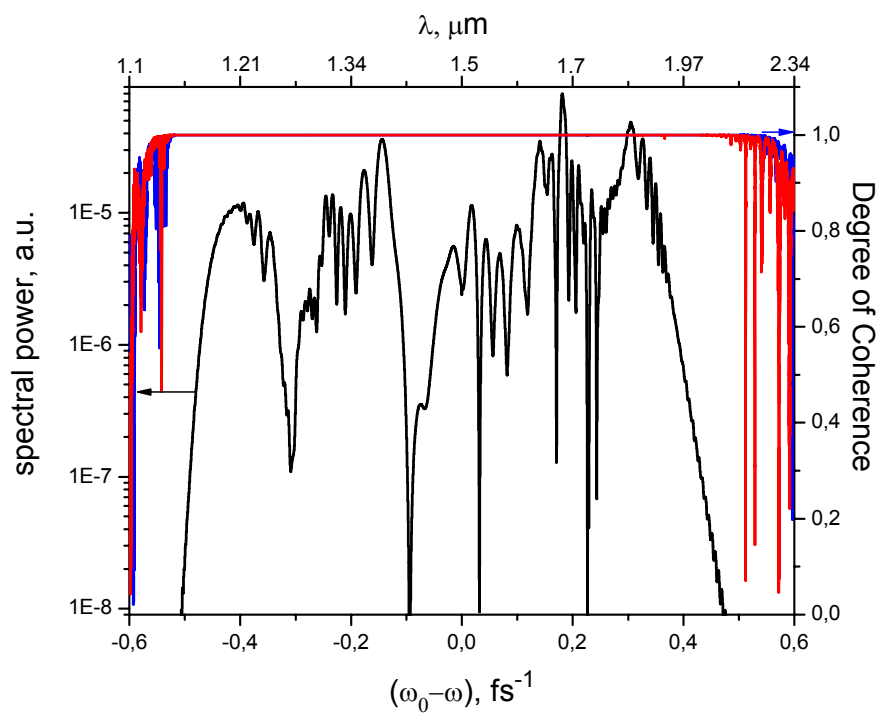

Fig. 2. Logarithm of spectral power and degree of coherence (blue curve) for SF6 fiber pumped by $40 \mathrm{GW} / \mathrm{cm}^{2} 60 \mathrm{fs} \mathrm{pulse}$ from Cr:YAG laser. The red curve corresponds to the coherence in absence of Raman interaction.

\section{References}

[1] J. Herrmann, U. Griebner, N. Zhavoronkov, A. Husakou, D. Nickel, J. C. Knight, W. J. Wadsworth, P. St. J. Russell, G. Korn, "Experimental Evidence for Supercontinuum Generation by Fission of Higher-Order Solitons in Photonic Fibers", Phys. Rev Lett., 88, 173901 (2002).

[2] E. Sorokin, S. Naumov, I.T. Sorokina, "Ultrabroadband infrared solid-state lasers", IEEE JSTQE 11, 690-712 (2005) .

[3] V. R. K. Kumar, A. K. George, W. H. Reeves, J. C. Knight, P. S. J. Russell, F. G. Omenetto, and A. J. Taylor, "Extruded soft glass photonic crystal fiber for ultrabroad supercontinuum generation", Opt. Express 10, 1520 (2002).

[4] V.L. Kalashnikov, E. Sorokin, S. Naumov, I.T. Sorokina, V.V. Ravi Kanth Kumar, A.K. George, "Low-threshold supercontinuum generation from an extruded SF6 PCF using a compact $\mathrm{Cr}^{4+}$ :YAG laser", Appl. Phys. B 79, 591 (2004).

[5] E. Sorokin, V.L. Kalashnikov, I.T. Sorokina, "The Raman effects in supercontinuum generation in highly nonlinear microstructured fibers at $1.5 \mu \mathrm{m}$ ", Proc. Advanced Solid-State Photonics Topical Meeting, January 29-February 1, 2006, USA, MB9.

[6] S.J. Carter, "Quantum theory of nonlinear fiber optics: pfase-space representations", Phys. Rev. A 51, 3274 (1995).

[7] K.L. Corwin, N.R. Newbury, J.M. Dudley, S. Coen, S.A. Diddams, B.R. Washburn, K. Weber, R.S. Windeler, "Fundamental amplitude noise limitations to supercontinuum spectra generated in a microstructured fiber", Appl. Phys. B 77, 269 (2003). 